\title{
Validating pore size estimates in a complex microfiber environment on a human MRI system
}

\author{
Chu-Chung Huang ${ }^{1,2}$ (D) | Chih-Chin Heather Hsu, ${ }^{3,4}$ | Feng-Lei Zhou ${ }^{5,6}$ | \\ Slawomir Kusmia $^{5,7,8}$ | Mark Drakesmith ${ }^{7}$ | Geoff J. M. Parker ${ }^{5,9,10}$ | Ching-Po Lin ${ }^{4}$ | \\ Derek K. Jones ${ }^{7}$ iif
}

${ }^{1}$ Key Laboratory of Brain Functional Genomics (MOE \& STCSM), Affiliated Mental Health Center (ECNU), Institute of Cognitive Neuroscience, School of
Psychology and Cognitive Science, East China Normal University, Shanghai, China
${ }^{2}$ Shanghai Changning Mental Health Center, Shanghai, China
${ }^{3}$ Center for Geriatrics and Gerontology, Taipei Veterans General Hospital, Taipei, Taiwan
${ }^{4}$ Institute of Neuroscience, National Yang Ming Chiao Tung University, Taipei, Taiwan
${ }^{5}$ Centre for Medical Image Computing, Department of Computer Science, University College London, London, United Kingdom
${ }^{6}$ School of Pharmacy, University College London, London, United Kingdom
${ }^{7}$ Cardiff University Brain Research Imaging Centre (CUBRIC), Cardiff University, Cardiff, United Kingdom
${ }^{8}$ Epilepsy Society MRI Unit, Chalfont St Peter, United Kingdom
${ }_{9}^{9}$ Department of Neuroinflammation, Queen Square Institute of Neurology, University College London, London, United Kingdom
${ }^{10}$ Bioxydyn Limited, Manchester, United Kingdom

\section{Correspondence}

Derek K. Jones, Cardiff University Brain

Research Imaging Centre (CUBRIC),

Cardiff University, Maindy Road, Cardiff,

CF24 4HQ, UK.

Email: jonesd27@cardiff.ac.uk

\section{Funding information}

EPSRC, Grant/Award Number: EP/ M029778/1; Wolfson Foundation; Wellcome Trust, Grant/Award Number: 096646/Z/11/Z and 104943/Z/14/Z; Biomedical Research Centre (BRC); University of Manchester; University College London; Shanghai Municipal Science and Technology Major Project: 2018SHZDZX01; ZJ Lab; Shanghai Center for Brain Science and BrainInspired Technology; Ministry of Science and Technology (MOST), Grant/Award Number: MOST 110-2321-B-010-004, MOST 110-2321-B-010-007, MOST 110-2634-F-010-001 and MOST 108-2321B-010-010-MY2
Purpose: Recent advances in diffusion-weighted MRI provide "restricted diffusion signal fraction" and restricting pore size estimates. Materials based on co-electrospun oriented hollow cylinders have been introduced to provide validation for such methods. This study extends this work, exploring accuracy and repeatability using an extended acquisition on a $300 \mathrm{mT} / \mathrm{m}$ gradient human MRI scanner, in substrates closely mimicking tissue, that is, non-circular cross-sections, intra-voxel fiber crossing, intra-voxel distributions of pore-sizes, and smaller pore-sizes overall.

Methods: In a single-blind experiment, diffusion-weighted data were collected from a biomimetic phantom on a $3 \mathrm{~T}$ Connectom system using multiple gradient directions/diffusion times. Repeated scans established short-term and long-term repeatability. The total scan time (54 min) matched similar protocols used in human studies. The number of distinct fiber populations was estimated using spherical deconvolution, and median pore size estimated through the combination of CHARMED and AxCaliber3D framework. Diffusion-based estimates were compared with measurements derived from scanning electron microscopy.

Results: The phantom contained substrates with different orientations, fiber configurations, and pore size distributions. Irrespective of one or two populations within the

Correction added after online publication 17 May, 2021. In accordance with the journal ‘s Author Guidelines, the statement of equal authorship has been moved to the Acknowledgments.

This is an open access article under the terms of the Creative Commons Attribution License, which permits use, distribution and reproduction in any medium, provided the original work is properly cited.

(C) 2021 International Society for Magnetic Resonance in Medicine 
voxel, the pore-size estimates $(\sim 5 \mu \mathrm{m})$ and orientation-estimates showed excellent agreement with the median values of pore-size derived from scanning electron microscope and phantom configuration. Measurement repeatability depended on substrate complexity, with lower values seen in samples containing crossing-fibers. Samplelevel repeatability was found to be good.

Conclusion: While no phantom mimics tissue completely, this study takes a step closer to validating diffusion microstructure measurements for use in vivo by demonstrating the ability to quantify microgeometry in relatively complex configurations.

\section{K E Y W O R D S}

crossing fiber, diameter, diffusion MRI, electron microscopy, microstructure, phantom

\section{1 | INTRODUCTION}

Obtaining reliable quantitative tissue microstructure information using non-invasive MRI has long been a holy grail in microstructural MRI. ${ }^{1,2}$ Improvements in gradient hardware $^{3,4}$ give increased sensitivity to smaller water displacements, and higher signal-to-noise ratio (SNR) per unit time, while improvements in modeling ${ }^{2}$ can potentially yield higher specificity to compartment-specific properties. Two measures of particular interest are: (1) the fraction of the signal that comes from spins trapped within pores, known as the "restricted signal fraction"5; and (2) the inner-diameter (pore-size) of restricting geometries. ${ }^{6,7}$ In white matter, for example, the former is taken as an indicator of "axon density," while the latter is taken as an indicator of axon diameter, one of the major factors influencing the speed of action potentials. $^{8,9}$

Most previous validations of such measurements have estimated diameters in tightly controlled architectures (eg, using a phantom comprising synthetic fibers all with the same orientation, or in the mid-line of the corpus callosum where the fibers are largely co-aligned) on small-bore preclinical scanners (exploiting the strong gradients that typically accompany such systems). ${ }^{7,10-18}$ In comparison, there is much less work validating measurements in more complex substrates using MRI systems designed for clinical use, which is essential for pushing forward in vivo microstructural quantification in human tissue.

Ex vivo/post-mortem brain samples might be the most direct route for validation since, by definition, they reflect the real physical complexity of the tissue, albeit with limitations imposed by changes in relaxation times, diffusivities, and tissue shrinkage. ${ }^{19}$ However, lack of parametric control over tissue properties, such as size, shape, or distribution makes the systematic study of the performance characteristics of a microstructural pipeline more challenging.

The ability to specify the microstructural properties of a substrate a priori can potentially facilitate the design of far more efficient experiments to ascertain accuracy and precision. Numerical/in silico phantoms have been used to simulate different substrates by modeling diffusion properties with different geometries. ${ }^{20,21}$ However, digital phantoms are generally over-simplistic in several respects, including the fact that they do not simulate acquisition conditions faithfully. ${ }^{22}$ To the best of our knowledge, this can only be achieved feasibly through actual scanning of physical phantoms comprising synthetic substrates. ${ }^{10,23-25}$ As discussed by Fan et al, ${ }^{10}$ while physical phantoms can never fully replace ex vivo samples in reflecting real tissue status, they serve as an important step between in silico simulations and actual construction of biological tissues.

Using a hollow textile filament (or "taxon") phantom, Fan et al, ${ }^{10}$ validated non-invasive pore size estimates on a human MRI system with ultra-strong gradients (up to $300 \mathrm{mT} / \mathrm{m}$ ). Sampling over a wide range of diffusion times and gradient strengths, they estimated inner diameter and restricted signal fraction using a simplification of the AxCaliber model ${ }^{7}$ that, as with ActiveAx, ${ }^{6}$ fits for a single pore diameter. Their results showed good agreement with the known phantom properties, supporting the feasibility of estimating microfiber pore size on a clinical MRI system. However, the dataacquisition took $38 \mathrm{~h}$, and the phantom comprised fiber with a: (1) single orientation; (2) circular cross-section; and (3) a single, relatively large (compared with diameters typically found in the brain ${ }^{26}$ ), diameter of $12 \mu \mathrm{m}$. While this work, therefore, represents an important step in understanding the capabilities of emerging hardware and modeling frameworks to recover microstructural parameters on a clinical system, it is important to extend the validation framework into more complex substrates, moving toward architectures that mimic tissue properties in vivo. Moreover, for full clinical translation, exploring the fidelity of microstructural estimates with shorter acquisition protocols is essential.

To approach the kinds of microstructural geometries found in vivo, and to achieve the parametric control of properties such as pore size, shape, density, and orientation, Zhou 
et al, ${ }^{27}$ developed the manufacture of co-electrospun microfibers from highly hydrophilic hollow polycaprolactone (PCL). Critically, this approach has a stochastic element, thereby introducing a distribution in the cross-sectional shape of individual pores, and facilitates control over pore size and orientation. ${ }^{25}$ This approach has been used to create "axonmimicking" fibers ${ }^{25,27}$ that resulted in anisotropic diffusion of water within them. Moreover, by tuning the fiber innerdiameter, the authors previously demonstrated control over diffusion tensor MRI-based properties such as fractional anisotropy and radial diffusivity ${ }^{25}$ and used these materials to help characterise signals from multidimensional diffusion encoding acquisitions. ${ }^{28}$

Again, while work such as described above represent steps toward validating estimates of pore size, several caveats remain. In previous validations of AxCaliber, the free diffusivities of the liquid and inner pore diameter were known a priori. Moreover, measurements were limited to samples with a single and known orientation (physical phantom/corpus callosum), limiting the generalizability of the validation of quantitative measurements across a whole organ, such as the brain. It is also important to ensure that such measurements have excellent short-term and long-term repeatability, to ensure that any observed changes in the signal/measurement reflect true changes in the substrate being imaged, rather than a perturbation introduced through noise/scanner instability/instabilities in the data-processing pipeline. To facilitate this, it is important to have a substrate that will not change its physical properties over time, but which also mimics the physical properties of the target substrate of interest (eg, white matter).

To address these issues, we aimed to validate AxCaliber estimations of microstructural parameters using coelectrospun substrates containing microfibers with unknown (to a subset of the authors) distributions of size, shape, and orientation, and complexity (ie, number of distinct compartment populations). This study was completed in a single-blind manner to prevent any potential bias in estimates, pre-processing or post hoc inference. Thus, an essential feature of this study was that any information on the phantom microstructure was totally withheld from a subset of the authors (C-CH, C-CHH, SK, DKJ) until all data acquisition, analysis, and final estimations were complete. To maintain translational relevance to in vivo applications, we used a diffusion-weighted imaging protocol with a total scan time less than $1 \mathrm{~h}$ (54 min). Constrained spherical deconvolution (CSD) ${ }^{29}$ was used to estimate the number of distinct fiber orientations and a single-parameter continuous Poisson distribution model ${ }^{30}$ within the Axcaliber $3 \mathrm{D}^{31}$ framework to fit the range of pore sizes in the biomimetic phantom.

\section{2 | METHODS}

\section{1 | Experimental design}

At the beginning of this single-blind validation study, a subset of the authors knew only that the phantom contained six tubes with hollow microfibers and one tube with pure liquid medium produced according to the method of Zhou et al. ${ }^{27,32,33}$ Other than a numbering system to reference each tube, it was impossible to differentiate between the samples with the naked eye. The construction of the phantom is shown in Figure 1A, and "sagittal" and "axial" sections of the phantom through a diffusion-weighted MRI (dMRI) are shown in Figure 1B. Figure 1C shows a schematic overview of the experimental design, and full protocol details are described in the following sections.

\section{2 | Phantom construction and fiber characterization}

The co-electrospinning setup used to generate the phantom has been detailed previously. ${ }^{34}$ Briefly, a coaxial spinneret with two concentric needles was filled with a solution of 10 wt.\% PCL ( $\mathrm{Mn}=80 \mathrm{k} \mathrm{g} / \mathrm{mol})$ with $1 \mathrm{wt}$.\% polysiloxanebased surfactant (PSi) in $\mathrm{CHCl}_{3} / \mathrm{DMF}(8 / 2 \mathrm{w} / \mathrm{w}$ ) (outer needle, inner diameter $=1.19 \mathrm{~mm}$ ) and $4 \mathrm{wt} . \%$ polyethylene oxide (PEO, Mv $=900 \mathrm{~kg} / \mathrm{mol}$ ) in water (inner needle, inner diameter $=0.41 \mathrm{~mm}) .{ }^{27}$ The outer needle was connected to the positive electrode of a DC voltage power supply, while the fiber collector was grounded. A voltage of 9 or $15 \mathrm{kV}$ was applied to generate uniaxially or randomly aligned fibers, respectively. A rotating drum (diameter $=11 \mathrm{~cm}$ ), spinning at 800 or 10 revolutions/min, was placed at a distance of $\sim 5$ or $14 \mathrm{~cm}$ from the tip of the concentric needles as a collector. To allow the fiber deposition to be spread uniformly, the collector was positioned on a translational $x-y$ stage, moving left and right at $1 \mathrm{~mm} / \mathrm{s}$. The flow rates of the outer and inner solutions were fixed at 3 and $1 \mathrm{~mL} / \mathrm{h}$, respectively. The translation distance of the $x-y$ stage was 30 or $55 \mathrm{~mm}$ for uniaxially or randomly aligned fibers.

Six phantom samples labeled 1-6 were constructed by packing one (tubes 1, 5, and 6) or two blocks (tubes 2, 3, and 4) comprising $\sim 24$ fiber layers $(10 \mathrm{~mm} \times 10 \mathrm{~mm})$ into a $15 \mathrm{~mL}$ centrifuge tube filled with deionized water. The same co-electrospun substrate was used for the construction of tubes 1 and 6 , where the fibers comprising the block were stacked in an interleaved fashion, crossing at $45^{\circ}$ (tube 1) and $90^{\circ}$ (tube 6); fibers comprising the blocks in tube 2 and 3 were randomly oriented; fibers within each block inside tubes 4 and 5 were uniaxially aligned $\left(0^{\circ}\right)$, but in tube 4 , 
(A)

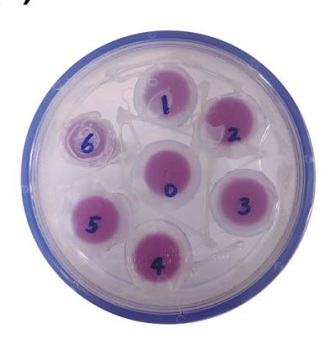

(B)

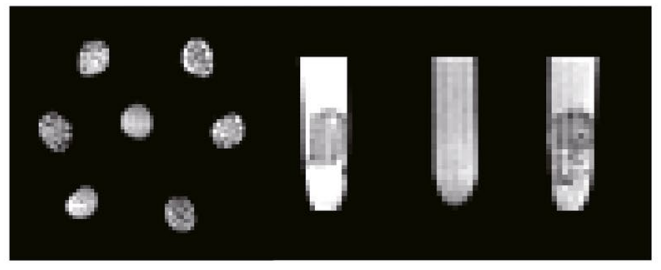

(C)

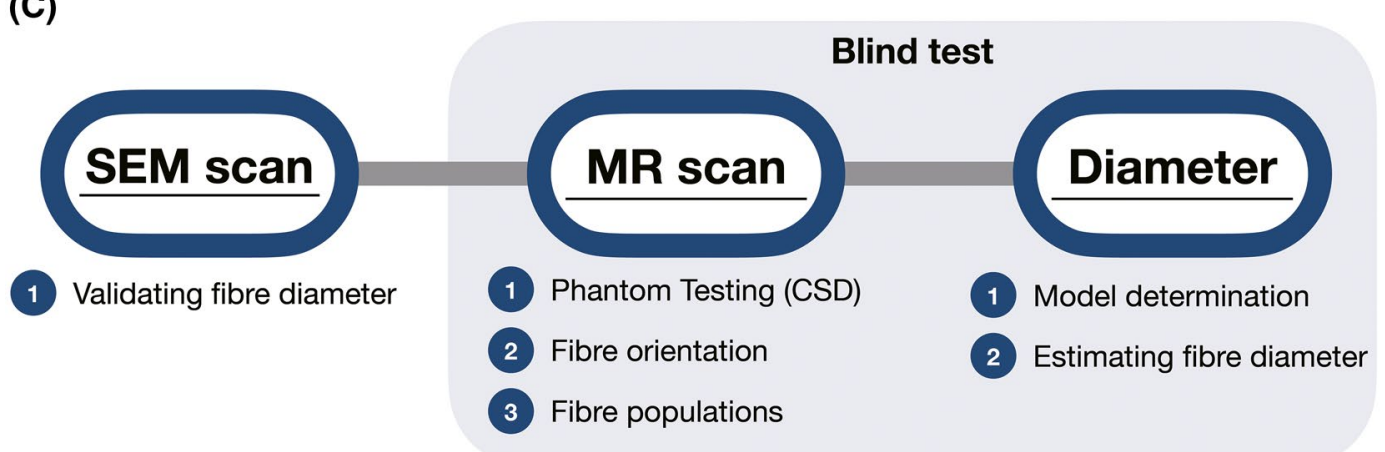

FIGURE 1 The overview of the diffusion phantom and the design of the single-blind experiment. A, There were seven tubes in the phantom container, each tube held one or two block-samples of fiber phantom with liquid filled (the characteristic of filling medium was unknown during experiments). B, The sagittal and coronal slices of the diffusion-weighted image of the phantom are shown to demonstrate the voxels with anisotropic phantoms. $\mathrm{C}$, The flowchart describes the design of the single-blind experiment

the two distinct blocks (made from the same substrate) were oriented at $90^{\circ}$ to each other. Six phantom samples were initially placed into a vacuum-degassing chamber to remove air bubbles before they were assembled into a cylindrical plastic container (inner diameter: $\sim 140 \mathrm{~mm}$; height: $\sim 180 \mathrm{~mm}$ ); The six tubes (1-6) were spaced equally around the circumference of the container and one tube containing only deionized water (labeled 0) was placed at the center. Due to the single-blind study design, the specification/configuration of the phantom was not revealed until the MRI acquisition and data analyses were complete.

\section{3 | MRI experiment}

The diffusion-weighted phantom scans were performed on the 3T Connectom MRI system (maximum gradient strength $=300 \mathrm{mT} / \mathrm{m}$ ) using a Siemens 32-channel head coil at the Cardiff University Brain Research Imaging Centre (CUBRIC). The phantom container was placed along the scanner's y-axis (vertical orientation and perpendicularly to the static magnetic $\mathrm{B}_{0}$ field), so that any air bubbles floated to the top of the tubes, and was immobilized with cushions to minimize vibrations during scanning.

The same imaging protocol was applied four times to evaluate the repeatability of diameter estimates. The first two scans (scan 1 and scan 2) were conducted on May 20,
2019, and June 3, 2019, whereas the other two scans (scan $3 \mathrm{a}$ and scan 3b) were a pair of immediate scan-rescan acquisitions conducted on June 16, 2020. All scans were performed under ambient conditions and the temperature was not recorded. The protocol comprised two diffusion frameworks, the Composite Hindered And Restricted ModEl of Diffusion (CHARMED) ${ }^{5}$ and the AxCaliber $3 D$ framework. ${ }^{31}$ The CHARMED model considered the diffusion signal to arise from a combination of hindered and restricted diffusion components and fitted the data to the composite model, with a fixed diameter distribution of fiber to estimate signal fractions, diffusivity parameters, and axonal orientations. In contrast, the AxCaliber model expands CHARMED by introducing the diameter distribution of restricted cylindrical fibers as an unknown function to estimate but, in its original implementation, only considers diffusion-encoding along a single axis, assumed to be orthogonal to the fiber orientation.

By combining CHARMED and AxCaliber, AxCaliber $3 D$ enables axon diameter distributions to be recovered for more complicated fiber configurations, and with arbitrary orientation of the fibers with respect to the diffusion encoding. Both datasets were acquired using a diffusion-weighted spinecho blipped-CAIPI (EPI) sequence ${ }^{35}$ with $1.5 \mathrm{~mm}$ isotropic resolution, with parameters summarized in Table 1. For the CHARMED acquisition, the diffusion gradient pulse duration and the diffusion time were both fixed, and the gradient amplitude was varied between $51 \mathrm{mT} / \mathrm{m}$ and $281 \mathrm{mT} / \mathrm{m}$ 
TAB L E 1 Diffusion-weighted image protocol

\begin{tabular}{|c|c|c|c|c|c|}
\hline b-Values $\left(\mathrm{s} / \mathrm{mm}^{2}\right)$ & $\begin{array}{l}\text { No. of diffusion } \\
\text { directions }\end{array}$ & $\begin{array}{l}\text { Gradient Strength } \\
(\mathrm{mT} / \mathrm{m})\end{array}$ & $\begin{array}{l}\text { Range of q-value } \\
\left(\mu \mathrm{m}^{-1}\right)\end{array}$ & $\begin{array}{l}\text { Gradient pulse } \\
\text { duration }(\delta, \mathrm{ms})\end{array}$ & $\begin{array}{l}\text { Diffusion } \\
\text { times }(\Delta, \mathrm{ms})\end{array}$ \\
\hline \multicolumn{6}{|c|}{ CHARMED $(\mathrm{TR} / \mathrm{TE}=4500 / 74 \mathrm{~ms})$} \\
\hline 200 & 20 & 51.30 & 0.015 & \multirow[t]{6}{*}{7} & \multirow[t]{6}{*}{24} \\
\hline 500 & 20 & 81.12 & 0.024 & & \\
\hline 1200 & 30 & 125.7 & 0.038 & & \\
\hline 2400 & 61 & 177.7 & 0.053 & & \\
\hline 4000 & 61 & 229.4 & 0.068 & & \\
\hline 6000 & 61 & 281.0 & 0.084 & & \\
\hline \multicolumn{6}{|c|}{ 3D-AxCaliber $(\mathrm{TR} / \mathrm{TE}=5000 / 138 \mathrm{~ms})$} \\
\hline $2200 / 4400$ & \multirow[t]{6}{*}{30 for each shell } & $200.1 / 283.0$ & $0.060 / 0.084$ & \multirow[t]{6}{*}{7} & 18 \\
\hline $3600 / 7150$ & & $204.0 / 287.5$ & $0.061 / 0.086$ & & 27 \\
\hline $4900 / 9800$ & & $203.7 / 288.1$ & $0.061 / 0.086$ & & 36 \\
\hline $6200 / 12400$ & & $203.5 / 287.9$ & $0.061 / 0.086$ & & 45 \\
\hline $8400 / 16750$ & & $203.8 / 287.9$ & $0.061 / 0.086$ & & 60 \\
\hline $12750 / 25500$ & & $203.6 / 288.0$ & $0.061 / 0.086$ & & 90 \\
\hline
\end{tabular}

resulting in b-values ranging from 200 to $6000 \mathrm{~s} / \mathrm{mm}^{2}$. In each shell, the diffusion-encoding gradient directions were uniformly distributed over the unit sphere according to Jones et al. ${ }^{36}$ For AxCaliber3D, images with six different diffusion times were acquired using a fixed gradient pulse duration and varying the prescribed b-value between 2200 and 25500 $\mathrm{s} / \mathrm{mm}^{2}$, with a maximal gradient amplitude of $288 \mathrm{mT} / \mathrm{m}$. In each b-value shell, data were acquired over 30 uniformly distributed encoding directions. A total of $24 \mathrm{~b}=0 \mathrm{~s} / \mathrm{mm}^{2} \mathrm{im}-$ ages were interleaved between the different $b$-shells to allow for the correction of signal drift. The total acquisition time was $54 \mathrm{~min}$. Other imaging parameters included a field-ofview of $128 \mathrm{~mm}$ x $128 \mathrm{~mm}, 30$ continuous slices, with an isotropic voxel size of $1.5 \mathrm{~mm}$, simultaneous multi-slice factor of 2, partial Fourier of 6/8, and no GRAPPA was applied.

\section{4 | Data pre-processing and analysis}

The regions of interest (ROIs) used for model fitting were selected manually from the cross-sectional images of the tubes through the following steps: (1) thresholding the $b=0$ $\mathrm{s} / \mathrm{mm}^{2}$ images with intensity higher than $10 \%$ of its maximal signal intensity to avoid processing background noise; (2) separating the thresholded binary mask image spatially into nine ROI components for each fiber sample in the tubes (labeled as tubes 1, 2a, 2b, 3a, 3b, 4a, 4b, 5, 6, corresponding to the label on the phantom tubes as shown in Figure 1A); (3) cropping the ROI along the axis parallel to the tube orientation to ensure that it only covered the anisotropic samples in the tube; (4) Eroding the ROIs by two voxels to eliminate inhomogeneous partial-volume voxels at the boundaries between the phantom material and the plastic tube containing the material.

For both the CHARMED and AxCaliber3D datasets, the signal pre-processing involved: (1) denoising ${ }^{37} ;(2) \mathrm{drift}$ correction $^{38}$; (3) eddy current distortion ${ }^{39}$; (4) gradient nonlinearity distortion ${ }^{40}$; and (5) correction for Gibbs-ringing artifacts. $^{41}$

The $\mathrm{b}=2400 \mathrm{~s} / \mathrm{mm}^{2}$ (61 directions) shell data of the preprocessed CHARMED data were used to derive the fiber orientation distribution function (fODF) via CSD with $1_{\max }=8$ in MRtrix3 (http://www.mrtrix.org/). The number of unique fODF peaks in each sample was extracted via Newton optimization. ${ }^{42}$ The threshold was set to 0.1 absolute amplitude of the fODF and above $33 \%$ of the maximum amplitude of each voxel to exclude small peaks. ${ }^{43}$

The AxCaliber3D framework, ${ }^{31}$ embedded into the Microstructure Diffusion Toolbox (MDT, https://github.com/ robbert-harms/MDT), was used to estimate the microfiber inner diameters. MDT includes a model-cascade approach ${ }^{44}$ that shortens the overall run time and improves fitting accuracy and precision. Initially, CHARMED data were used to model the signal using one hindered diffusion compartment (using a zeppelin diffusion tensor) and one or two restricted diffusion compartments (based on the estimated number of fiber populations from the CSD analysis) using van Gelderen's ${ }^{45}$ expression for restricted diffusion in a cylinder. The estimated fiber peak orientations then served as prior fixed parameters and initial starting estimates of the restricted diffusion signal fractions for the fitting of the AxCaliber3D model. The total measured signal decay was assumed to be a sum of diffusion-weighted signal decays for each pore size weighted by their respective area-weighted probability and 
the pore-size populations were modeled with a continuous Poisson diameter distribution, ${ }^{30}$ yielding an average pore-size for each voxel in the ROI.

\section{5 $\quad$ Scanning electron microscopy of the phantom}

The surface morphology and cross-sections of co-electrospun fibers were observed using a FEI Quanta 650 field emission gun scanning electron microscope (SEM) with an accelerating voltage of $5 \mathrm{kV}$. The co-electrospun fiber specimens were coated with a gold-palladium film to increase their conductivity and the fiber strips were cut using a sharp scalpel in liquid nitrogen for imaging their cross-sections. ImageJ (imagej.nih.gov/ij) was used to measure the pore size (fiber inner diameters) using its "Pore Measurement" function. For each sample, pore sizes (areas) were automatically measured from five different SEM images and manually converted into the fiber inner diameters within the sample with the assumption of circular pores. ${ }^{33}$ The area-weighted fiber inner diameters and fractions were calculated using a method reported previously. ${ }^{46}$ Those responsible for the phantom manufacture (F.Z., G.P.) noted that, because fiber deposition could not be controlled precisely during the co-electrospinning process, some large "extra-fiber" pores were formed randomly and frequently in the phantom (see Figure 5 and Supporting Information Figure S1, which is available online). In these spaces, for the diffusion times used here, the spins would not experience hindrance/restriction during their displacement, effectively leading to a third mode of diffusion, that is, "free water." Estimating all pore sizes, irrespective of dimension (and including these larger pores) would give a false impression of restricting pore size, and so the estimated restricting fiber volume fractions from SEM were estimated by dividing the total area of pores with diameter less than $15 \mu \mathrm{m}$, by the total area of all pores (ie, pores with diameters in the range: $[0, \infty])$, see Table 2$)$.

TA B LE 2 Phantom properties estimated by dMRI and SEM

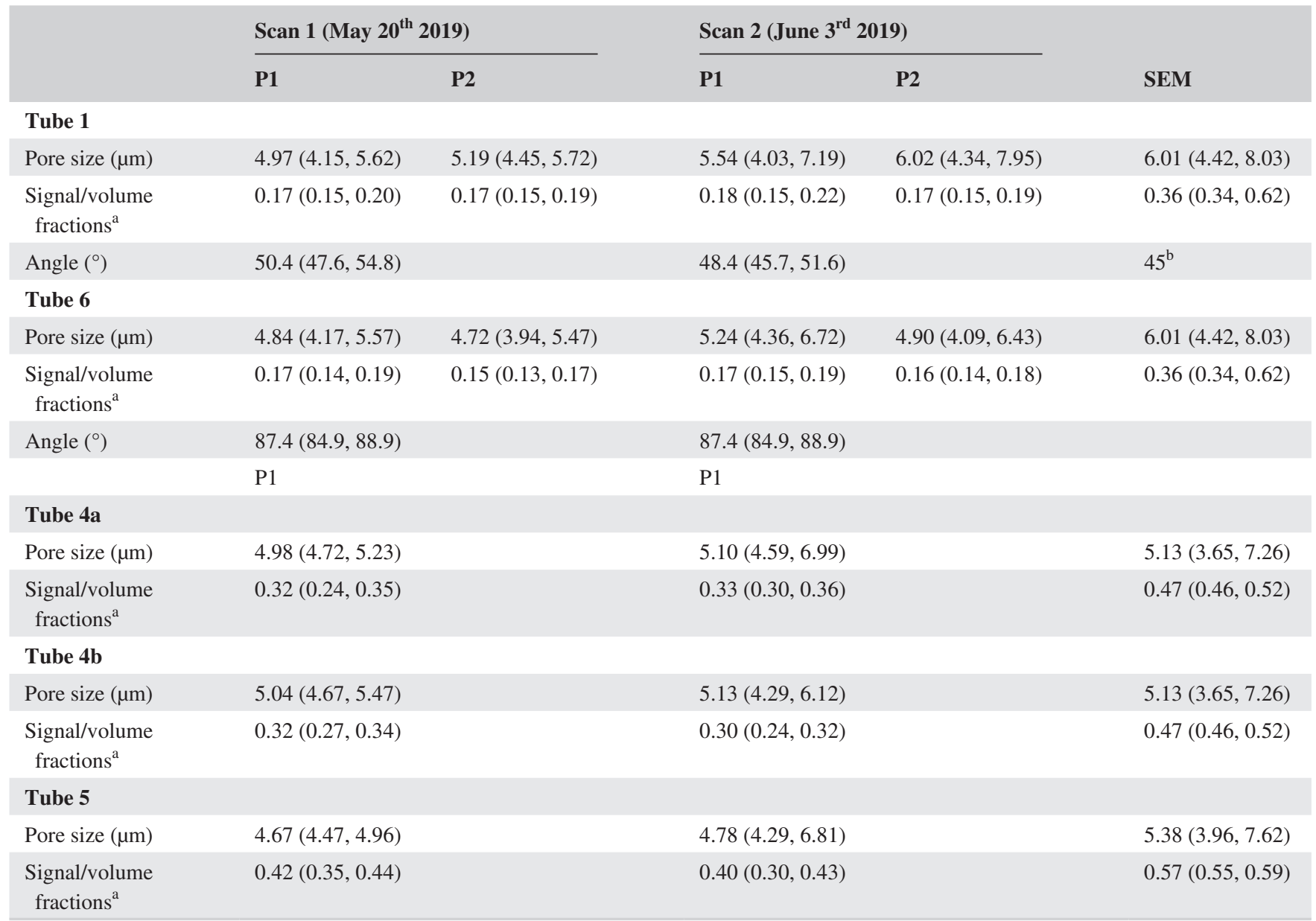

Note: Parameter estimates for the different tubes. The median (and upper and lower quartiles) are shown. "Angle" represents the crossing angle between two fiber populations. Note, tube 1 and tube 6 contain crossing fibers so parameters for each population (P1 and P2) are shown separately.

${ }^{\text {a }}$ The estimated signal fraction from dMRI represents the fitting result of restricted diffusion signal fraction, while the volume fraction of SEM represents the ratio of the total area of pores with diameter $\leq 15 \mu \mathrm{m}$ to the total area of pores with diameter in the range $[0, \infty])$.

${ }^{\mathrm{b}}$ The fibers in the blocks of tubes 1 and 6 were designed to be interleaved, crossing at $45^{\circ}$ and $90^{\circ}$. 


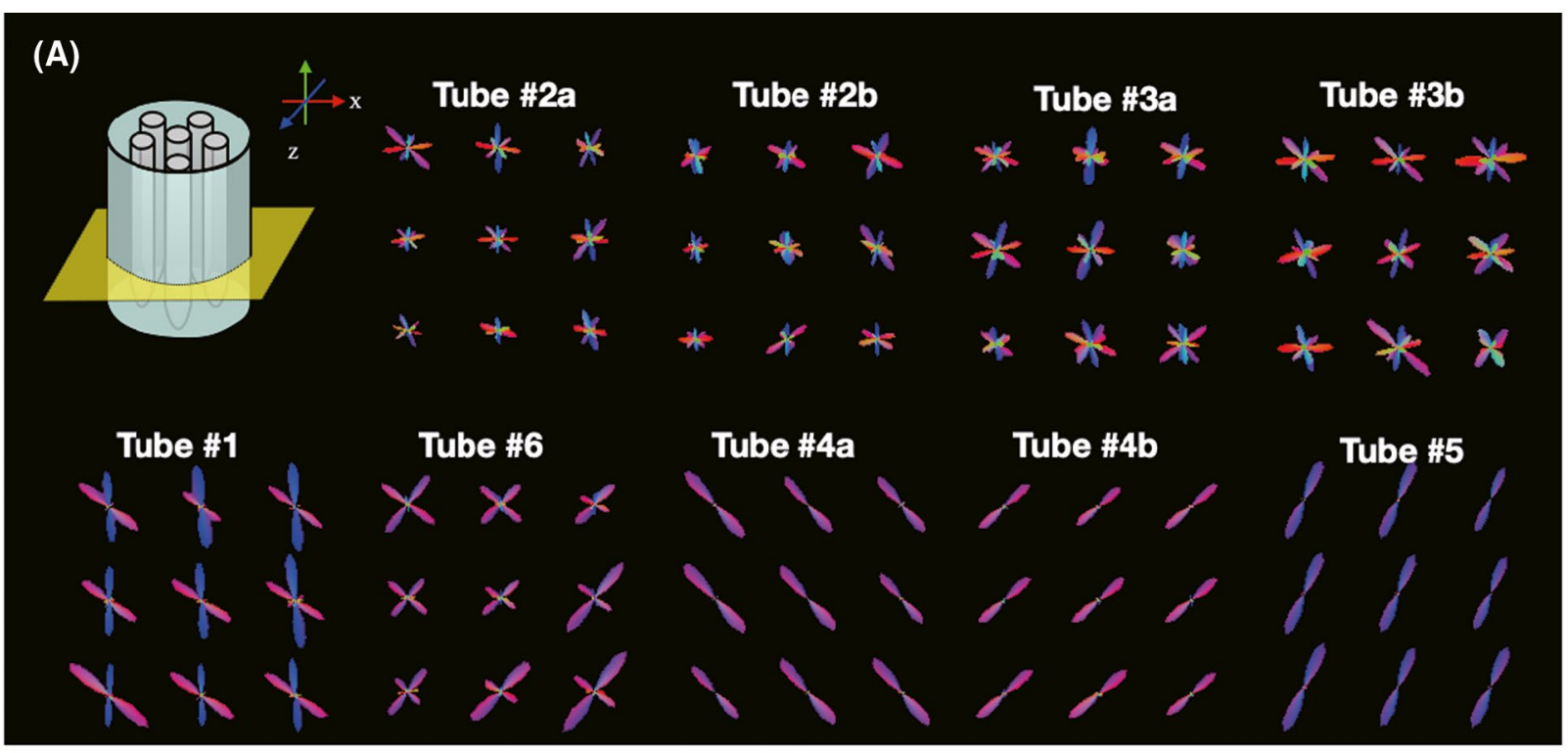

(B)

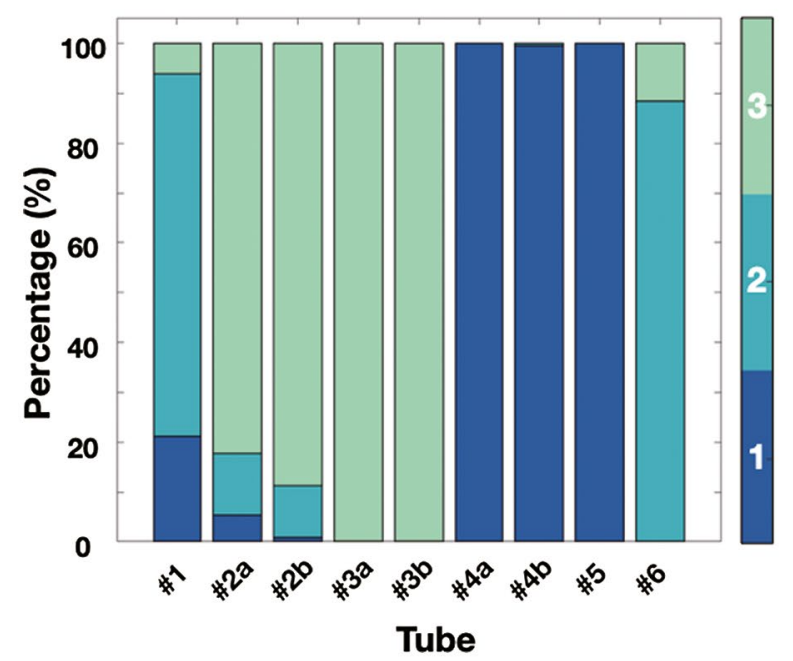

(C)

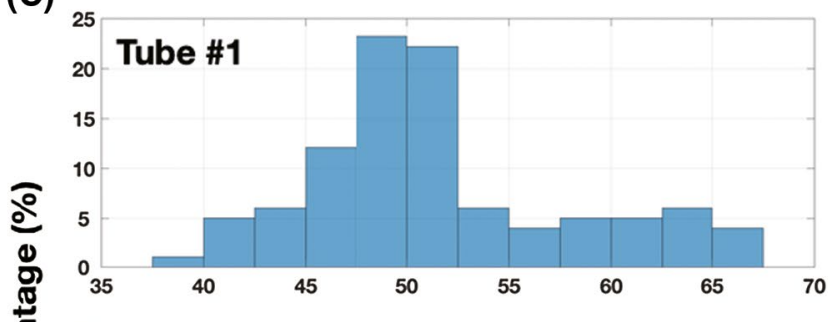

F I G U RE 2 The representative measurement from the first scan on May 20,2019. Measurement of the fODF in the anisotropic phantom obtained from CSD. The fiber orientations are shown with directional colour encoding in x-z plane (A) and x-y plane (B) view. As the figure shows, tubes 1 and 6 contain crossing fibers, tubes $4 \mathrm{a}, 4 \mathrm{~b}, 5$ contain a single orientation, while tubes $2 \mathrm{a}, 2 \mathrm{~b}, 3 \mathrm{a}$, and $3 \mathrm{~b}$ appear to contain randomly oriented fibers. $\mathrm{C}$, The number of unique fODF peak orientations in each sample voxel with the threshold of 0.1 absolute amplitude and $33 \%$ of the maximum amplitude. The color represents the number of unique peaks, where blue $=1$, cyan $=2$, and green represent 3 or more peaks. $\mathrm{D}$, The histogram of the estimated angle between fibers in each voxel in tubes 1 and 6 (left and right, respectively)

\section{6 | Statistical analysis}

Due to the different positioning of the phantom between scans 1,2 , and 3 , it was not possible to obtain exact spatial correspondence between the ROIs across the different scans to establish long-term repeatability. Therefore, rather than comparing estimates on a voxel-by-voxel basis, we evaluated repeatability of the pore-size estimate distribution using the Kolmogorov-Smirnov test (KS-test) and the Jensen-Shannon distance. To compare the distributions obtained from the dMRI scans and the ground truth from SEM, we first binned the accumulated area fraction of fiber pore size into 30 bins (bin range from 0 to $15 \mu \mathrm{m}$, bin width $=$ $0.5 \mu \mathrm{m})$. We then calculated the median value, and the first and third quantiles (Q1 and Q3) to identify asymmetric distributions. A two-sample KS-test determined whether the two samples (SEM versus dMRI, or between the repeat-scan data) came from the same continuous distribution. The intraclass correlation coefficient (ICC), within-voxel coefficient of variation $(\mathrm{CV})$, and the repeatability coefficient (RC) were calculated to evaluate the repeatability of measures at both the voxel-level and sample-level of the repeat-scan. ${ }^{47}$ 


\section{3 | RESULTS}

\section{1 | Phantom examination using dMRI}

\subsection{1 $\quad$ SNR evaluation}

The SNR was calculated as the mean signal within the tubes ( $\geq 10 \%$ of maximal intensity in $\mathrm{b}=0 \mathrm{~s} / \mathrm{mm}^{2}$ image) divided by the SD of the background $(<10 \%$ of maximal intensity in $\mathrm{b}=0 \mathrm{~s} / \mathrm{mm}^{2}$ image) using the non-diffusion-weighted $(\mathrm{b}=$ $0 \mathrm{~s} / \mathrm{mm}^{2}$ ) image. The SNRs for the four scans were: May 20, $2019=45.3$, June 3, $2019=44.7$, first scan on June 16, 2020 $=34.2$, second scan on June $16,2020=34.2$.

\subsection{2 | Fiber population information, based on CSD model}

Due to the single-blind study design, the number of distinct fiber populations in each phantom tube not known before analysis. Since the number of restricted-diffusion compartments is set a priori in the CHARMED model, it was therefore necessary to first estimate the number of distinct fiber populations, for which CSD was used. Figure 2 shows the CSD-estimated fiber orientations in the axial and sagittal plane of the tubes. Four distinct phantom configurations were observed: (1) randomly oriented fibers; (2) single-oriented fiber-population; (3) two fiber-populations; (4) high diffusivity, isotropic medium (in the central tube, later revealed to be water). Both tube 1 and tube 6 showed an obvious crossing pattern, where the proportions of voxels that contained two distinct fiber populations were 0.73 and 0.88 , respectively. No distinct anisotropic characteristic was observed in tube 2 and tube 3, in which the fODF analysis suggested that more than $80 \%$ of voxels contained more than three distinct populations in each voxel. For the purposes of our study, we assumed this was consistent with a random distribution and not amenable to analysis with the CHARMED/AxCaliber3D frameworks. Moreover, we observed that tube 4 contained two distinct fiber substrate blocks (one on top of the other); thus, we labeled these two blocks as tube $4 \mathrm{a}$ and tube $4 \mathrm{~b}$ for further analyses and reporting.

\subsection{3 | Angle information, based on CHARMED}

The median crossing angles of tube 1 and tube 6 were estimated using the CHARMED framework to be $50.37^{\circ}$ and $87.36^{\circ}$, respectively (Figure $2 \mathrm{C}$ and Table 2 ). No crossing fiber configuration was observed in tube $4 a$, tube $4 \mathrm{~b}$, or tube 5 (Figure 2A-B).

\subsection{4 | Pore sizes, based on 3D-AxCaliber}

Due to parameter explosion, voxels with a large number of randomly aligned fibers (eg, tubes 2 and 3 ) are not amenable to analysis by the Axcaliber3D framework. Thus, we only reported fiber diameter estimates in samples identified as containing one or two fiber orientations (tubes $1,4 \mathrm{a}, 4 \mathrm{~b}, 5$, and 6). Table 2 shows the estimated median fiber diameters and the estimated restricted signal fraction. In tube 1 (deemed to contain two distinct fiber-populations), the estimated median diameter for population $1(\mathrm{p} 1)$ was $4.97 \mu \mathrm{m}$, and population 2 (p2) was $5.19 \mu \mathrm{m}$ (mean diameter: $\mathrm{p} 1 / \mathrm{p} 2=4.96 / 5.09 \mu \mathrm{m}$ ). Tube 4 was deemed to contain two blocks, each with a single fiber-population, but with distinct orientations. The median pore diameter in the first block (4a) was $4.98 \mu \mathrm{m}$, and in the second block (4b) was $5.04 \mu \mathrm{m}$ (mean diameter: $4 \mathrm{a} / 4 \mathrm{~b}$ $=5.00 / 5.05 \mu \mathrm{m})$. Tube 5 was deemed to contain a singlefiber-population model, with a median diameter of $4.67 \mu \mathrm{m}$ (mean diameter $=4.77 \mu \mathrm{m})$. Finally, tube 6 was also deemed to contain two distinct fiber populations (median diameter: $\mathrm{p} 1 / \mathrm{p} 2=4.84 / 4.72 \mu \mathrm{m}$; mean diameter: $\mathrm{p} 1 / \mathrm{p} 2=4.89 / 4.76$ $\mu \mathrm{m})$. The fittings and parameter estimates were homogeneous across most voxels within the ROI (Figure 3 ).

\subsection{5 | Comparing the dMRI-derived estimates with SEM-derived estimates}

The authors responsible for manufacturing the phantom (F.Z., G.P.) confirmed that two of the six tubes contained orientationally disperse samples (tubes 2 and 3), two contained samples with two fiber populations with crossing angles (tubes 1 and 6), two tubes contained single fiber population blocks (tubes 4 and 5), and one contained purely isotropic media (water). Figure 2A shows that the estimated orientations of the fiber populations were consistent with the ground truth fiber configuration; the median of crossing angles of tube 1 and tube 6 were $50.37^{\circ}$ and $87.36^{\circ}$ (Figure $2 \mathrm{C})$, respectively, relative to the ground truth values of $45^{\circ}$ and $90^{\circ}$ (see the Discussion section regarding the precision of SEM/manufacturing). Figure 3 shows the Poisson fitting for all voxels within each ROI to recover the median diameter inside the fiber phantom across each scan. As noted above, due to the manufacturing process, the samples in tubes 1 and 6 , and tubes $4 \mathrm{a}$ and $4 \mathrm{~b}$ were derived from the same substrate; thus, there was only one set of SEM images available for each pair. For tubes 1 and 6, tubes $4 a$ and $4 \mathrm{~b}$, and tube 5 , the median (mean) diameters derived from SEM were 6.01 (6.43), 5.13 (5.82), and 5.38 (5.92) $\mu \mathrm{m}$, respectively (Table 2 ). The dMRI-derived restricted signal fractions are similar to the values derived from SEM (total area of pores with diameter $\leq 15 \mu \mathrm{m} /$ total area of pores with diameter in the range $[0, \infty])$, in which tube 1 
(A) Tube \#1
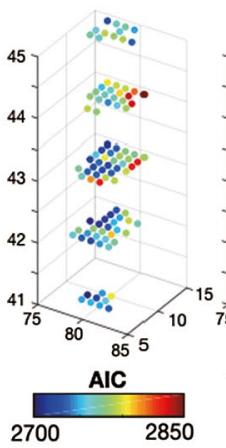

(C) Tube \#4a

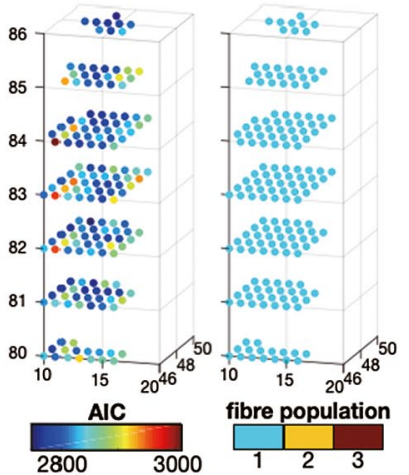

(E) Tube \#5

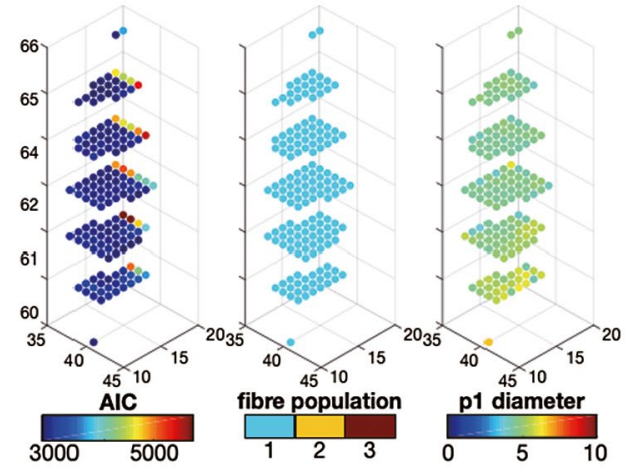

(B) Tube \#6

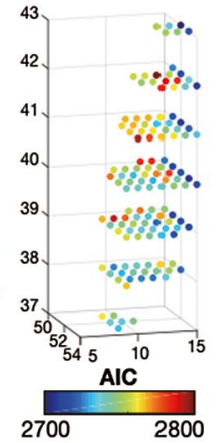

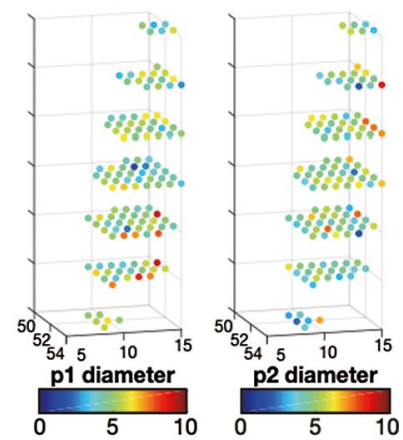

(D) Tube \#4b

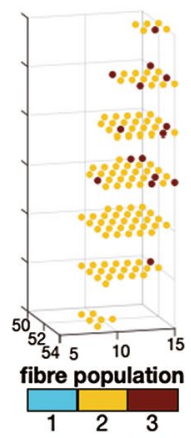

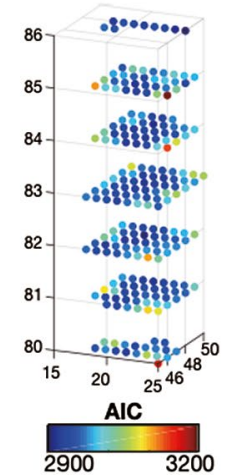
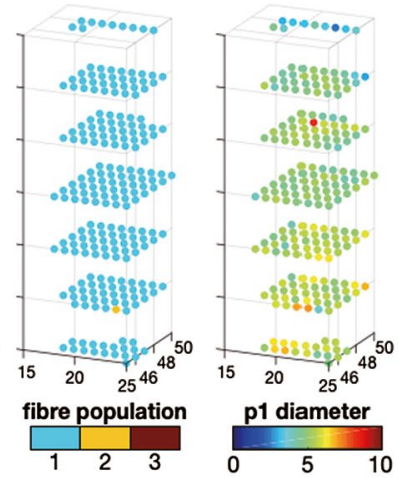

(F) Tubes in $\mathrm{BO}$ image
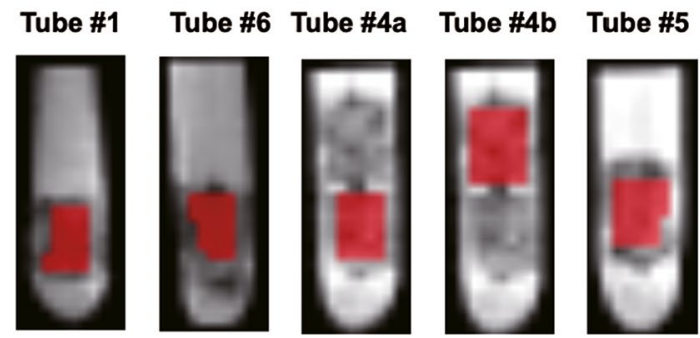

F I G URE 3 Fitting quality of fiber diameter estimations in five phantoms. To ensure fitting quality across different voxels, we examined three parameters including the Akaike Information Criterion (AIC), the number of distinct fiber populations ("fiber population"), and the pore size diameter ("p1 diameter"). This figure shows the manually selected ROI in the $2 \mathrm{D}$ image slice, and the fitting results in the 3D scatter plots in a voxel-wise manner. Samples containing two distinct fiber populations (A,B); samples with a single fiber population (C,D)

and tubes 6,4 , and 5 are $0.36,0.47$, and 0.57 , respectively (Shown in Table 2).

The KS-test indicated that the distributions recovered from the first dMRI scan and SEM were significantly different $(P<.01)$. No significant differences were found between dMRI and SEM results in scan 2, scan 3a, and scan 3b, except for tube $4 \mathrm{a}$ in scan $3 \mathrm{~b}$ (Figure 4 and Table 3 ). The phantom characterization in different tubes is presented by the SEM micrographs (see Figure 5A-C) in which we observed that the phantom does contain some "extra-fiber"-like spaces. The histogram of pore size diameter against the areaweighted fraction was also shown in Figure 5D to depict the distribution of pore sizes in different phantom blocks.

\subsection{6 | Repeatability of the dMRI- derived estimates}

Table 3 shows the KS-test applied to different scans among different tubes for evaluating the long-term and short-term 


\section{(A) Scan 1}

Tube \#1 \& \#6

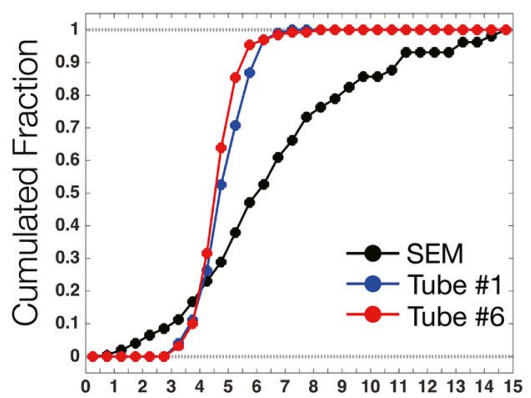

Tube \#4_1 \& \#4_2

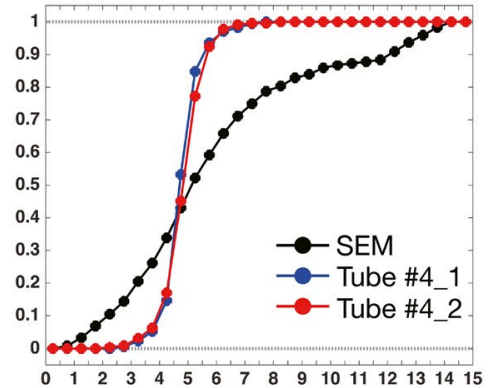

Tube \#5

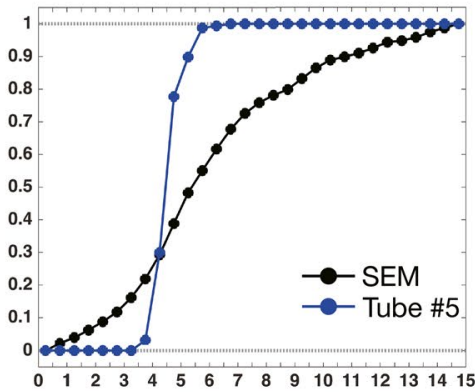

\section{(B) Scan 2}
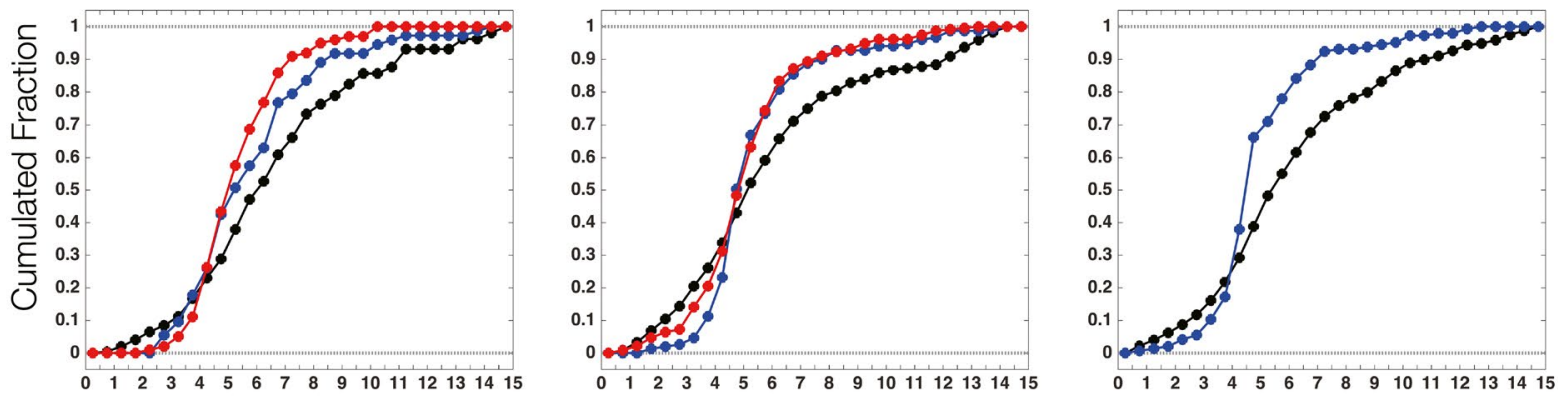

\section{(C) Scan 3_1}
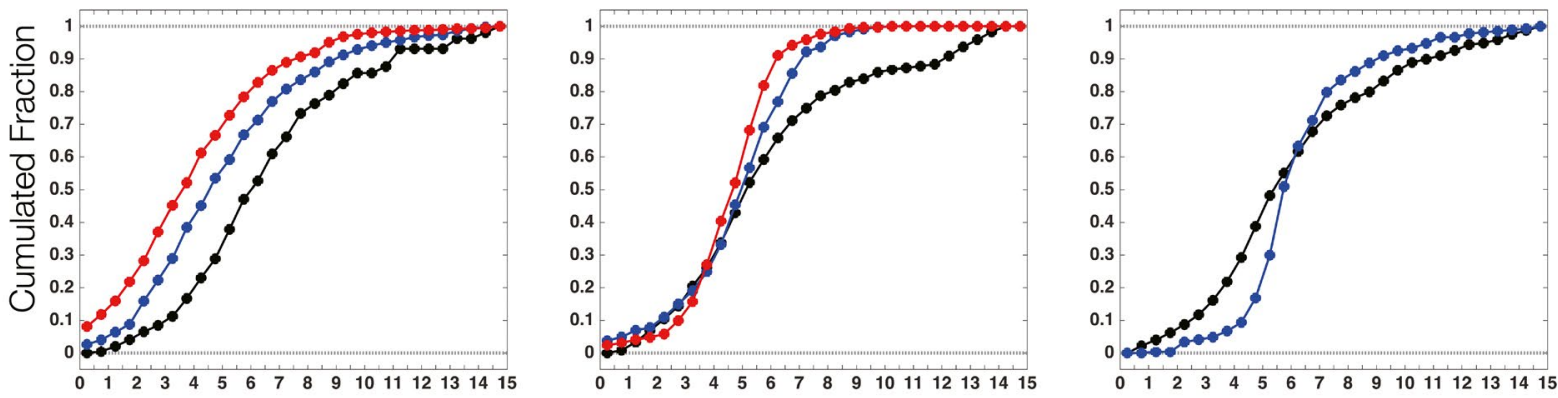

\section{(D) Scan 3_2}

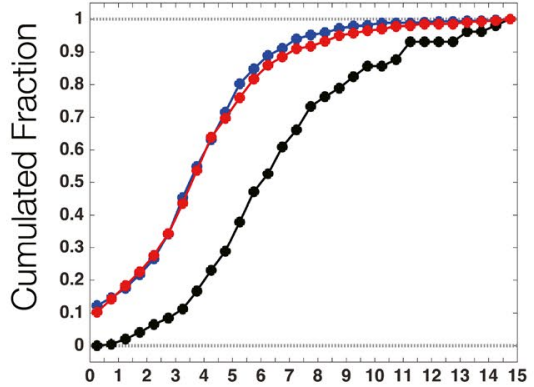

Pore Size Diameter ( $\mu \mathrm{m})$

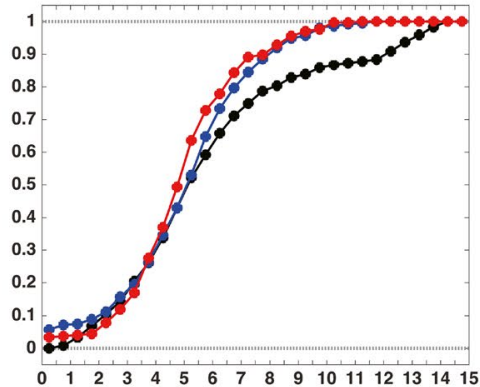

Pore Size Diameter $(\mu \mathrm{m})$

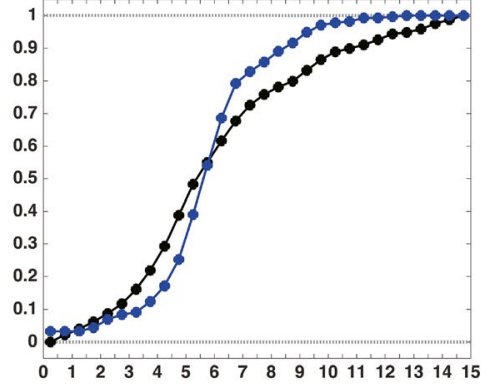

Pore Size Diameter $(\mu \mathrm{m})$

F I G URE 4 Cumulated fractions of the estimated fiber diameters from MRI and SEM. This figure shows the Poisson fitting for all voxels within each ROI for the different scans. The ground truth distribution of the pore size (estimated by SEM) is shown by the black line in each panel. Rows A to D represent the result from the different scans. The left column shows the two-fiber-population of tube 1 and tube 6 , the middle column shows the single-fiber-population of tube $4 \mathrm{a}$ and $4 \mathrm{~b}$, and the right column shows the single fiber-population of tube 5 


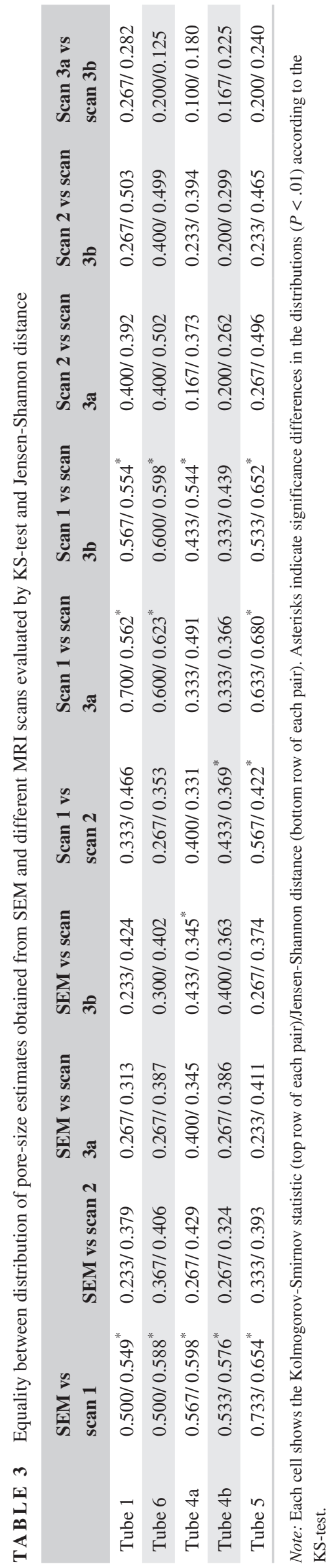

repeatability. The distribution obtained from scan 1 is significantly different to those obtained from later scans, where all recovered distributions are broadly similar. The experimental design of the third scan session (ie, no phantom repositioning between the two scans) allowed estimates of short-term repeatability on a voxel-wise basis (Figure 6 and Table 4). For tube $4 a$ and tube $4 b$, the voxel-level RC values of the estimated diameters are $4.13 \mu \mathrm{m}$ and $4.10 \mu \mathrm{m}$, the ICC values are 0.519 and 0.361 , respectively. The tubes containing samples with crossing fiber architectures (tubes 1 and 6) showed higher RC (tube 1/tube 6: $5.52 \mu \mathrm{m} / 4.43 \mu \mathrm{m}$ ) and lower ICC values between repeat scans (ICC tube 1/tube 6: 0.150 / 0.131). The sample-level RC value is $1.13 \mu \mathrm{m}$, the ICC value is 0.727 , and the $\mathrm{CV}$ value is $11.3 \%$.

\section{4 | DISCUSSION}

This single-blind study used a 3T Connectom human MRI scanner, advanced modeling, and a co-electrospun hollow PCL-PSi microfiber phantom to establish the reliability of microfiber diameter estimates in a scan time $<1 \mathrm{~h}$.

The results demonstrate fiber orientation and median pore size estimates that are highly comparable with results obtained by SEM, demonstrating the validity and robustness of the microstructural imaging pipeline with the phantom configuration used here. Compared with others in the literature, this phantom confers several advantages. The inner diameter approximates the median of the range of diameters within the human white matter $(0.25 \sim 10 \mu \mathrm{m})^{48}$ (although please see the Limitations section below). Moreover, the pore shape is more comparable to that seen in vivo making the phantom more "biomimetic" than other phantoms developed to date. Finally, (see Figure 5), the substrate contains larger extrafiber "voids" between the restricting geometries (a result of the manufacturing process) where the diffusion path-length will be considerably longer than for spins trapped within the intra-fiber pores. Thus, the phantom has surrogate "extrafiber" compartments as well as intra-fiber compartments, which again pushes the properties closer to that of real tissue.

A previous study used a phantom comprising both extraand intra-fiber compartments with a uniform inner diameter $(12 \pm 0.9 \mu \mathrm{m}) .{ }^{10}$ The same group recently constructed a phantom with an inner diameter of $0.8 \mu \mathrm{m},{ }^{14}$ and have started to fashion cross-fiber configurations. However, no quantitative estimates of pore-size in these more complex configurations have been reported. Validation of such estimates in phantoms with complex architectures is necessary since human white matter fiber bundles are not perfectly co-aligned, even in "single fiber" populations, and $60 \sim 90 \%$ of voxels contain multiple fiber orientations. ${ }^{42}$ Thus, the acquired signal in each voxel may originate from the restricted water across several fiber bundles in different orientations or even more 


\section{(A) Tube \#1 and Tube \#6}
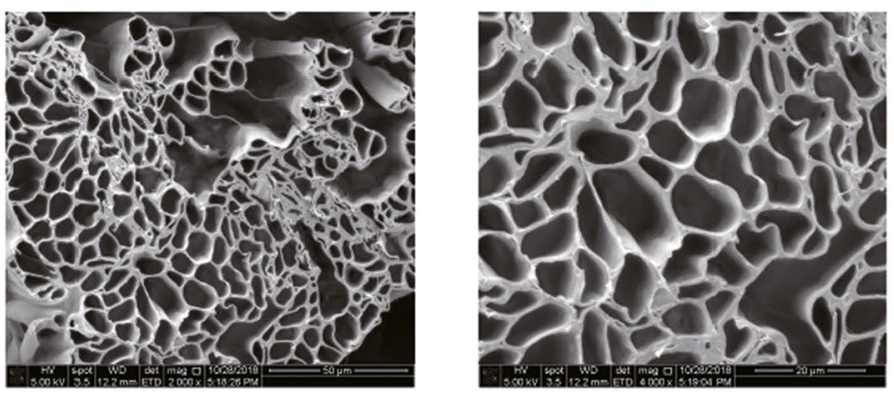

\section{(B) Tube \#4a and Tube \#4b}
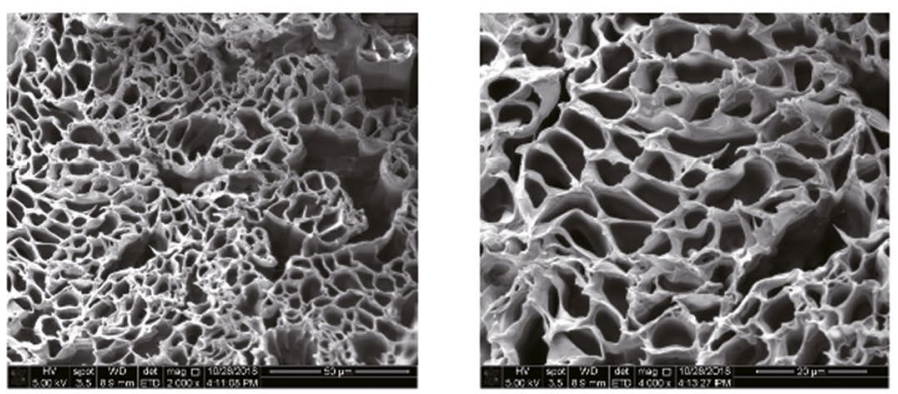

\section{(C) Tube \#5}
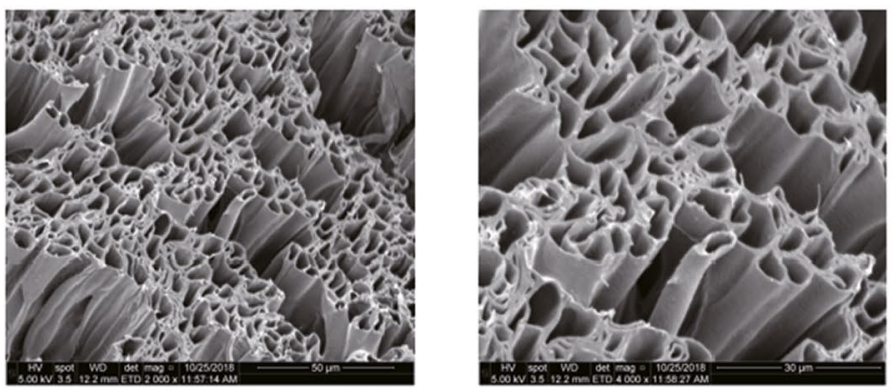

(D)

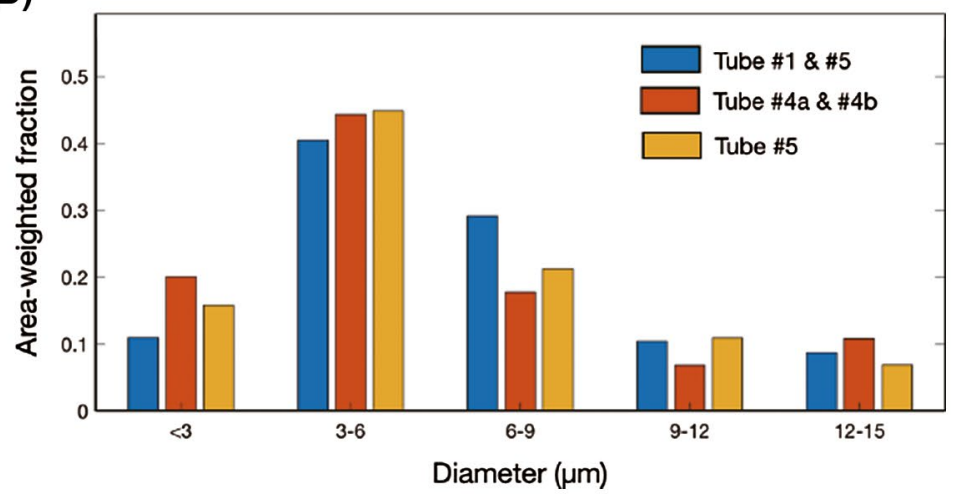

F I G URE 5 SEM images of

Co-Electrospun PCL-Psi fiber phantom.

A-C, SEM images with low (left) and higher (right) resolution. D, The area-weighted fractions of each sample pore size are shown in blue (tube 1 and tube 6), orange (tube 4a and $4 \mathrm{~b}$ ), and yellow (tube 5) complicated geometrical configurations (eg, axonal diameter), increasing uncertainty in fiber orientation estimates. ${ }^{49}$ Such partial volume effects confound the estimation of fractional anisotropy and fiber diameter in restricted volumes.
The present study extends previous work by validating the AxCaliber3D framework using a more sophisticated phantom that includes a range of pore sizes (with a median around 5 $\mu \mathrm{m}$ ), and both single and crossing fiber-orientations (about 


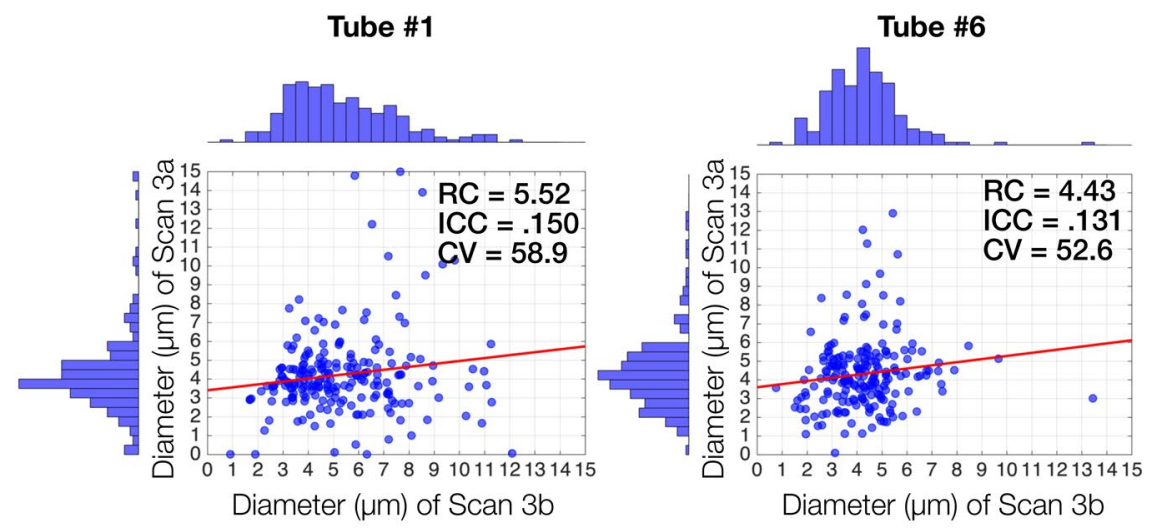

Tube \#4a

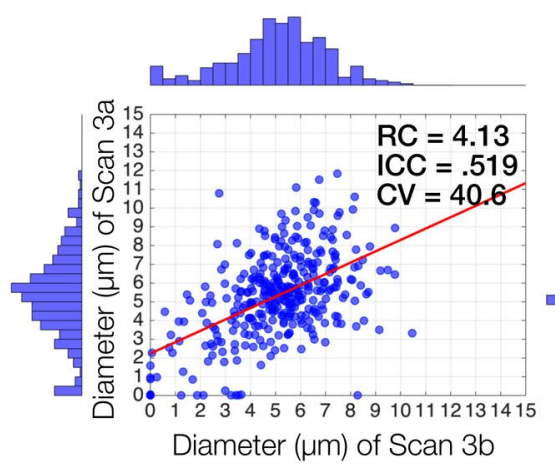

Tube \#4b

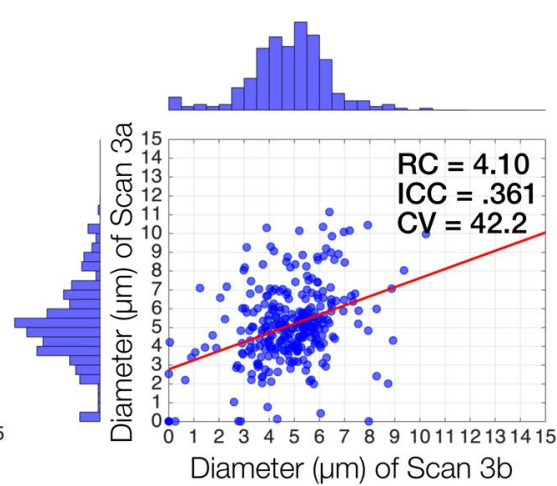

Tube \#5

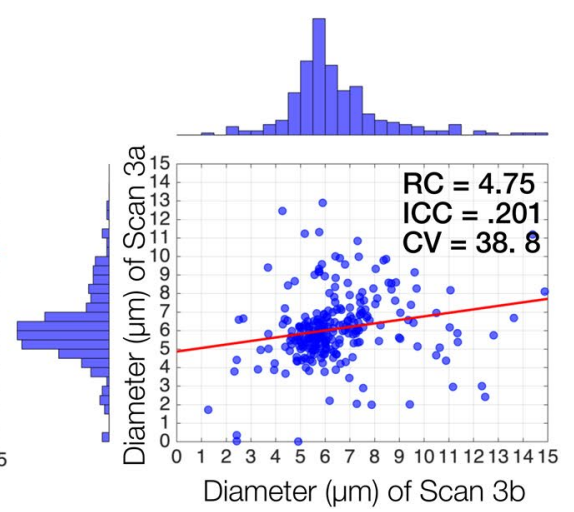

F I G U RE 6 The ICC between immediate scan and rescan. The upper row plots show the estimated diameter from scan $3 \mathrm{a}$ against that from scan $3 \mathrm{~b}$ of block samples with crossing orientation (in tube 1 and 6), whereas the bottom row plots are block samples with single orientation (in tube 4 and 5). The line of best fit between the data from the repeated scans is shown in red. RC, repeatability coefficient ( $\mu \mathrm{m}$ ); CV: within-voxel coefficient of variance $(\%)$

TA B LE 4 Repeatability between different MRI scans 3a and scan $3 b$

\begin{tabular}{|c|c|c|c|}
\hline & $\begin{array}{l}\text { RC } \\
(\mu \mathrm{m})\end{array}$ & ICC & $\mathrm{CV}(\%)$ \\
\hline \multicolumn{4}{|l|}{ Voxel-level repeatability } \\
\hline Tube 1 & 5.52 & 0.150 & 58.9 \\
\hline Tube 6 & 4.43 & 0.131 & 52.6 \\
\hline Tube $4 \mathrm{a}$ & 4.13 & 0.519 & 40.6 \\
\hline Tube $4 b$ & 4.10 & 0.361 & 42.2 \\
\hline Tube 5 & 4.75 & 0.201 & 38.8 \\
\hline Sample-level repeatability & 1.13 & 0.727 & 11.3 \\
\hline
\end{tabular}

Note: Asterisks are shown to indicate that $P<.01$.

$45^{\circ}$ and $90^{\circ}$ ). Regarding non-crossing single fiber conditions, the AxCaliber framework has been shown to recover axonal diameter distributions accurately. ${ }^{7,50}$ In the current study, to account for crossing fiber configurations, we used AxCaliber $3 \mathrm{D}^{31}$ with a continuous Poisson pore size distribution $^{30}$ to resolve diameters. On the whole, regardless of whether the sample contained one or two fiber populations, the recovered fiber orientation and pore-size estimates agreed well with measurements obtained by direct SEM.
Good repeatability is critical to quantitative MRI research to provide stable metrics that are less influenced by measurement instability. Long-term repeatability facilitates the study of subtle longitudinal changes in pore size, while short- (and long-) term variability both impact the random errors and precision of the estimation model. Our results showed inconsistency of estimated pore-size distributions between the first scan session and other scan sessions. Across the longterm scans, it was not possible to perform a voxel-by-voxel comparison due to the difference in the phantom positioning. Furthermore, images with lower SNR may introduce uncertainties in orientation and restricted-diffusion signal fraction estimation and, thus, result in variations in pore-size distribution. ${ }^{51}$ That is, differences in estimated pore-size distribution might be explained, in part, by differences in the SNR.

We observed a reduction in SNR of approximately $25 \%$ over $1 \mathrm{y}$ (between scans 2 and 3), although no difference was observed between scans 1 and 2. The source of this variation in SNR is unclear but could possibly reflect changes in the phantom material. For example, in the co-electrospinning manufacturing process, the core solution is PEO in water while the shell solution is PCL in Chcl3+DMF. The hollow microfibers are formed in situ after the evaporation of the 
solvents in both the shell and core. The PEO polymer is assumed to deposit on the inner surface of the resultant hollow PCL fibers but is not removed before the phantom is assembled. It can be expected that PEO would dissolve gradually in water, when the hollow fibers are filled with water. However, PEO has a very high molecular weight $(900 \mathrm{~kg} / \mathrm{mol})$ and dissolves very slowly at room temperature. The PCL polymer in the microfiber shell is subject to hydrolytic degradation, taking 2-4 y for a complete degradation, depending on the initial molecular weight and surrounding fluid. ${ }^{52}$ Therefore, considering the 1-y gap between the second and third scan sessions, a certain level degradation of PCL polymer can be also expected in the water-filled phantom. This may have shortened $\mathrm{T}_{2}$, leading to a reduction in SNR and is worthy of further investigation, but is beyond the scope of the current work.

Duval et al, ${ }^{53}$ previously demonstrated stable AxCaliber estimates in the spinal cord of healthy human participants, with correlation coefficient (r) 0.64 . We note that in the spinal cord, the axons tend to be largely co-axial. In our study, pore-size estimates demonstrated good repeatability at the sample-level ( $\mathrm{ICC}=0.727, \mathrm{RC}=1.13 \mu \mathrm{m})$, whereas the repeatability of pore-size estimates at the voxel-level was considerably better for "single orientation" samples than for those containing multiple fiber orientations. However, the short-term repeatability remained poor in both cases, which suggests the uncertainty at the voxel level that may still be affected by the errors from many parameters fitting in the model or potential residual misalignment in the scan-rescan test. Nevertheless, pore size and fiber orientations were estimated accurately by the proposed framework, but the repeatability at the voxel level should be re-examined and improved in the future.

\section{1 | Limitations of the study and future directions}

While our work demonstrated the strength and reliability of AxCaliber3D model to resolve the complex fiber architectures in this particular substrate, it is important to keep in mind that this study also has several limitations. Most importantly, we caution against full extrapolation to "axon diameter" mapping. While this phantom is a definite move toward the white matter properties, it would be premature (and incorrect) to conclude that this work fully licenses claims about the validity of AxCaliber3D for estimating axon diameters in all of white matter.

The most obvious hurdle preventing these claims is that the pore sizes remain considerably larger than the modal ex vivo white matter axons in the human brain. Further work is needed to manufacture pores with a smaller internal diameter. Second, there was no explicit attempt to control the temperature of the scan room during data acquisition. Lack of temperature control may have led to differences in the diffusivities of the phantom substrate between scans which may have affected the precision/ repeatability of the microstructural estimates. Although we did not anticipate this to be a major contributor, future work should record the real-time temperature during scanning to clarify such a possible confound. Moreover, we observed changes in the diffusion MRI characteristics of the phantom materials between scans 1 and 2 that are challenging to explain. We considered degradation of the phantom, but the only potential evidence of this is a change of around $25 \%$ in SNR that was observed between scans 2 and 3, over a period of $1 \mathrm{y}$; this does not explain the differences between scans 1 and 2. Additionally, as can be seen in Figure 4 and Table 3, the cumulative histograms of fiber diameters for the SEM measurements of the phantom (measured before any of the MRI scans) match the cumulative histograms of fiber diameters from the diffusion MRI scans at time points 2, 3a, and $3 \mathrm{~b}$ well. The diffusion MRI cumulative histograms from scans $2,3 \mathrm{a}$, and $3 \mathrm{~b}$ also match each other well but do not match the diffusion MRI histograms from scan 1 . The cause of this outlier behaviour for the diffusion measurements at scan 1 requires further investigation in future longitudinal studies; at this time, we are unable to rule out potential short-term issues with water penetration into the phantom material, scanning temperature variations, or errors in the diffusion MRI data acquisition as potential causes.

Third, the phantom was not explicitly designed to mimic the relative size and shape of the extra-axonal space seen in tissue; thus, estimates of tortuosity and extra-axonal timedependence are unlikely to reflect the situation in vivo. ${ }^{12,30,54}$ Fourth, the degree to which water exchanges across the fiber membranes is currently unknown, although a reasonable degree of restriction is apparent in the clear presence of time-dependent diffusion. ${ }^{28}$ Finally, due to the way in which the phantom is manufactured, the substrate is heterogeneous; thus, the control of the "ground truth" fiber angle and pore size is imprecise, which adds uncertainty to the cross-validation process. Thus, achieving perfect agreement between the dimensions extracted from the SEM of a subsample of the material and the sample used for imaging can be challenging.

Despite these limitations, this study can be considered as a useful step in the evolution of validating pore size estimates in complex geometries on a human MRI scanner. Similar validations in non-uniform pore phantoms in relatively short scanning times will enlighten the clinical practice of microstructural imaging in different living tissues including, but not limited to, the prostate, ${ }^{55}$ and muscle fibers. ${ }^{56,57}$

Owing to the blind nature of the experiment, the sample geometry and restricted diffusivity were totally unknown $a$ priori. Thus, a wide range of diffusion times (diffusion time: $19 \sim 90$ 
$\mathrm{ms}$ ) was used to maximize the sensitivity of diffusion displacement to possible pore sizes. ${ }^{58}$ If more information is known $a$ priori, the acquisition protocol could be optimized accordingly, including a reduction in total acquisition time. This is likely to lead to improved precision of microstructural parameter estimation and may, therefore, also improve repeatability.

In summary, by spanning multiple diffusion times and gradient strengths on an ultra-strong gradient scanner, we successfully estimated the fiber architectures that had expected pore sizes lower than $10 \mu \mathrm{m}$ (around $5 \mu \mathrm{m}$ ) in both single-aligned fiber populations and in populations of crossing fibers within a new biomimetic phantom with nonuniform cross-sections, which more closely mimics the white matter features than previously used simple geometric phantoms. Our microstructure measurements show good agreement with the new generation diffusion phantom and support validity for microstructure quantification of complex environment at the micron level. Future work is underway to validate pore-size estimates in phantoms with more crossing populations (including completely random), variable "extracellular" volume fractions and smaller non-uniform pore-size diameter than studied here.

\section{ACKNOWLEDGMENTS}

The data were acquired at the UK National Facility for In Vivo MR Imaging of Human Tissue Microstructure funded by the EPSRC (grant EP/M029778/1), and The Wolfson Foundation. DKJ and MD were both supported by a Wellcome Trust Investigator Award (096646/Z/11/Z) and a Wellcome Trust Strategic Award (104943/Z/14/Z). The authors thank Dr John Evans for his help to acquire the repeat-scan MRI data. The authors also thank Dr Robbert Harms for his assistance with embedding the pore-size estimation routine into the MDT toolbox. FLZ was supported by NIHR UCLH Biomedical Research Centre (BRC) grant and UK-MRC ImagingBioPro grant (MR/R025673/1). GJM Parker has a shareholding and part time appointment and directorship at Bioxydyn Ltd. which provides MRI services. GJM Parker also has a shareholding and directorship at Queen Square Analytics Ltd. which provides MRI services. The authors gratefully acknowledge the assistance from Prof. Julie E. Gough and Prof. Gareth R. Williams with access to lab space and electron microscopy facilities at The University of Manchester and University College London, respectively. CPL was supported by the Ministry of Science and Technology (MOST) of Taiwan (grants MOST 110-2321-B-010-004, MOST 110-2321-B-010-007, MOST 110-2634-F-010-001, and MOST 108-2321-B-010-010-MY2). We acknowledge the supports of Shanghai Municipal Science and Technology Major Project (No.2018SHZDZX01), ZJ Lab, and Shanghai Center for Brain Science and Brain-Inspired Technology. Author Chu-Chung Huang, Chih-Chin Heather Hsu and Feng-Lei Zhou contributed equally to this work.

\section{CONFLICT OF INTEREST}

GJM Parker has a shareholding and part time appointment and directorship at Bioxydyn Ltd. which provides MRI services. GJM Parker also has a shareholding and directorship at Queen Square Analytics Ltd. which provides MRI services.

\section{ORCID}

Chu-Chung Huang (DD https://orcid.

org/0000-0002-9096-9610

Chih-Chin Heather Hsu (ID) https://orcid.

org/0000-0003-1860-1034

Feng-Lei Zhou (D) https://orcid.org/0000-0002-8348-4658

Derek K. Jones (D) https://orcid.org/0000-0003-4409-8049

\section{REFERENCES}

1. Assaf Y, Pasternak O. Diffusion tensor imaging (DTI)-based white matter mapping in brain research: a review. J Mol Neurosci. 2008;34:51-61.

2. Novikov DS, Kiselev VG, Jespersen SN. On modeling. Magn Reson Med. 2018;79:3172-3193.

3. Jones DK, Alexander DC, Bowtell R, et al. Microstructural imaging of the human brain with a "super-scanner": 10 key advantages of ultra-strong gradients for diffusion MRI. Neuroimage. 2018;182:8-38.

4. McNab JA, Edlow BL, Witzel T, et al. The human connectome project and beyond: initial applications of $300 \mathrm{mT} / \mathrm{m}$ gradients. Neuroimage. 2013;80:234-245.

5. Assaf Y, Basser PJ. Composite hindered and restricted model of diffusion (CHARMED) MR imaging of the human brain. Neuroimage. 2005;27:48-58.

6. Alexander DC, Hubbard PL, Hall MG, et al. Orientationally invariant indices of axon diameter and density from diffusion MRI. Neuroimage. 2010;52:1374-1389.

7. Assaf Y, Blumenfeld-Katzir T, Yovel Y, Basser PJ. AxCaliber: a method for measuring axon diameter distribution from diffusion MRI. Magn Reson Med. 2008;59:1347-1354.

8. Drakesmith M, Harms R, Rudrapatna SU, et al. Estimating axon conduction velocity in vivo from microstructural MRI. Neuroimage. 2019;203:116186.

9. Waxman SG. Determinants of conduction velocity in myelinated nerve fibers. Muscle Nerve. 1980;3:141-150.

10. Fan Q, Nummenmaa A, Wichtmann B, et al. Validation of diffusion MRI estimates of compartment size and volume fraction in a biomimetic brain phantom using a human MRI scanner with $300 \mathrm{mT} / \mathrm{m}$ maximum gradient strength. Neuroimage. 2018;182:469-478.

11. Fan Q, Tian Q, Ohringer NA, et al. Age-related alterations in axonal microstructure in the corpus callosum measured by highgradient diffusion MRI. Neuroimage. 2019;191:325-336.

12. Lee HH, Fieremans E, Novikov DS. What dominates the time dependence of diffusion transverse to axons: intra- or extra-axonal water? Neuroimage. 2018;182:500-510.

13. Lee HH, Papaioannou A, Kim SL, Novikov DS, Fieremans E. A time-dependent diffusion MRI signature of axon caliber variations and beading. Commun Biol. 2020;3:354.

14. Pathak S, Schneider W, Zuccolotto A, et al. Diffusion ground truth quantification of axon scale phantom: Limits of diffusion MRI on 
7T, 3T and Connectome 1.0. In Proceedings of the 28th Annual Meeting of ISMRM. 2020. Abstract 0737.

15. Shemesh N, Ozarslan E, Adiri T, Basser PJ, Cohen Y. Noninvasive bipolar double-pulsed-field-gradient NMR reveals signatures for pore size and shape in polydisperse, randomly oriented, inhomogeneous porous media. J Chem Phys. 2010;133:044705.

16. Shemesh N, Ozarslan E, Basser PJ, Cohen Y. Detecting diffusiondiffraction patterns in size distribution phantoms using doublepulsed field gradient NMR: theory and experiments. J Chem Phys. 2010;132:034703.

17. Benjamini D, Elsner JJ, Zilberman M, Nevo U. Pore size distribution of bioresorbable films using a 3-D diffusion NMR method. Acta Biomater. 2014;10:2762-2768.

18. Benjamini D, Komlosh ME, Basser PJ, Nevo U. Nonparametric pore size distribution using d-PFG: comparison to s-PFG and migration to MRI. J Magn Reson. 2014;246:36-45.

19. Miller KL, McNab JA, Jbabdi S, Douaud G. Diffusion tractography of post-mortem human brains: optimization and comparison of spin echo and steady-state free precession techniques. Neuroimage. 2012;59:2284-2297.

20. Close TG, Tournier J-D, Calamante F, et al. A software tool to generate simulated white matter structures for the assessment of fibre-tracking algorithms. Neuroimage. 2009;47:1288-1300.

21. Leemans A, Sijbers J, Verhoye M, Van der Linden A, Van Dyck D. Mathematical framework for simulating diffusion tensor MR neural fiber bundles. Magn Reson Med. 2005;53:944-953.

22. Ginsburger K, Poupon F, Beaujoin J, et al. Improving the realism of white matter numerical phantoms: a step toward a better understanding of the influence of structural disorders in diffusion MRI. Front Phys. 2018;6:12.

23. Bach M, Fritzsche KH, Stieltjes B, Laun FB. Investigation of resolution effects using a specialized diffusion tensor phantom. Magn Reson Med. 2014;71:1108-1116.

24. Fieremans E, Lee HH. Physical and numerical phantoms for the validation of brain microstructural MRI: a cookbook. Neuroimage. 2018;182:39-61.

25. Hubbard PL, Zhou FL, Eichhorn SJ, Parker GJ. Biomimetic phantom for the validation of diffusion magnetic resonance imaging. Magn Reson Med. 2015;73:299-305.

26. Aboitiz F, Scheibel AB, Fisher RS, Zaidel E. Fiber composition of the human corpus callosum. Brain Res. 1992;598:143-153.

27. Zhou FL, Li Z, Gough JE, Hubbard Cristinacce PL, Parker GJM. Axon mimicking hydrophilic hollow polycaprolactone microfibres for diffusion magnetic resonance imaging. Mater Des. 2018; 137:394-403.

28. Lundell H, Nilsson M, Dyrby TB, et al. Multidimensional diffusion MRI with spectrally modulated gradients reveals unprecedented microstructural detail. Sci Rep. 2019;9:9026.

29. Tournier J-D, Yeh C-H, Calamante F, et al. Resolving crossing fibres using constrained spherical deconvolution: validation using diffusion-weighted imaging phantom data. Neuroimage. 2008;42:617-625.

30. De Santis S, Jones DK, Roebroeck A. Including diffusion time dependence in the extra-axonal space improves in vivo estimates of axonal diameter and density in human white matter. Neuroimage. 2016;130:91-103.

31. Barazany D, Jones DK, Assaf Y. AxCaliber 3D. In Proceedings of the 19th Annual Meeting of ISMRM. Montreal, Canada, 2011. Abstract 76.
32. Zhou FL, Hubbard PL, Eichhorn SJ, Parker GJ. Coaxially electrospun axon-mimicking fibers for diffusion magnetic resonance imaging. ACS Appl Mater Interfaces. 2012;4:6311-6316.

33. Zhou FL, Parker GJ, Eichhorn SJ, Hubbard Cristinacce PL. Production and cross-sectional characterization of aligned coelectrospun hollow microfibrous bulk assemblies. Mater Charact. 2015;109:25-35.

34. Zhou F-L, Hubbard PL, Eichhorn SJ, Parker GJM. Jet deposition in near-field electrospinning of patterned polycaprolactone and sugarpolycaprolactone core-shell fibres. Polymer. 2011;52:3603-3610.

35. Setsompop K, Cohen-Adad J, Gagoski BA, et al. Improving diffusion MRI using simultaneous multi-slice echo planar imaging. Neuroimage. 2012;63:569-580.

36. Jones DK, Horsfield MA, Simmons A. Optimal strategies for measuring diffusion in anisotropic systems by magnetic resonance imaging. Magn Reson Med. 1999;42:515-525.

37. Veraart J, Novikov DS, Christiaens D, et al. Denoising of diffusion MRI using random matrix theory. Neuroimage. 2016;142: 394-406.

38. Vos SB, Tax CMW, Luijten PR, et al. The importance of correcting for signal drift in diffusion MRI. Magn Reson Med. 2017;77:285-299.

39. Andersson JLR, Sotiropoulos SN. An integrated approach to correction for off-resonance effects and subject movement in diffusion MR imaging. Neuroimage. 2016;125:1063-1078.

40. Glasser MF, Sotiropoulos SN, Wilson JA, et al. The minimal preprocessing pipelines for the Human Connectome Project. Neuroimage. 2013;80:105-124.

41. Kellner E, Dhital B, Kiselev VG, Reisert M. Gibbs-ringing artifact removal based on local subvoxel-shifts. Magn Reson Med. 2016;76:1574-1581.

42. Jeurissen B, Leemans A, Tournier JD, Jones DK, Sijbers J. Investigating the prevalence of complex fiber configurations in white matter tissue with diffusion magnetic resonance imaging. Hum Brain Mapp. 2013;34:2747-2766.

43. Roine $\mathrm{T}$, Jeurissen $\mathrm{B}$, Perrone D, et al. Informed constrained spherical deconvolution (iCSD). Med Image Anal. 2015;24:269-281.

44. Harms RL, Fritz FJ, Tobisch A, Goebel R, Roebroeck A. Robust and fast nonlinear optimization of diffusion MRI microstructure models. Neuroimage. 2017;155:82-96.

45. van Gelderen P, DesPres D, van Zijl PC, Moonen CT. Evaluation of restricted diffusion in cylinders. Phosphocreatine in rabbit leg muscle. J Magn Reson B. 1994;103:255-260.

46. Grech-Sollars M, Zhou FL, Waldman AD, Parker GJM, Hubbard Cristinacce PL. Stability and reproducibility of co-electrospun brain-mimicking phantoms for quality assurance of diffusion MRI sequences. Neuroimage. 2018;181:395-402.

47. Raunig DL, McShane LM, Pennello G, et al. Quantitative imaging biomarkers: a review of statistical methods for technical performance assessment. Stat Methods Med Res. 2015;24:27-67.

48. Alexander DC. A general framework for experiment design in diffusion MRI and its application in measuring direct tissuemicrostructure features. Magn Reson Med. 2008;60:439-448.

49. Schilling KG, Janve V, Gao Y, et al. Histological validation of diffusion MRI fiber orientation distributions and dispersion. Neuroimage. 2018;165:200-221.

50. Assaf Y, Alexander DC, Jones DK, et al. The CONNECT project: combining macro- and micro-structure. Neuroimage. 2013;80:273-282. 
51. Chen G, Zhang P, Li KE, et al. Improving estimation of fiber orientations in diffusion MRI using inter-subject information sharing. Sci Rep. 2016;6:37847.

52. Woodruff MA, Hutmacher DW. The return of a forgotten polymer-polycaprolactone in the 21st century. Prog Polym Sci. 2010;35:1217-1256.

53. Duval T, Smith V, Stikov N, Klawiter EC, Cohen-Adad J. Scanrescan of axcaliber, macromolecular tissue volume, and g-ratio in the spinal cord. Magn Reson Med. 2018;79:2759-2765.

54. Sykova E, Nicholson C. Diffusion in brain extracellular space. Physiol Rev. 2008;88:1277-1340.

55. Lemberskiy G, Fieremans E, Veraart J, et al. Characterization of prostate microstructure using water diffusion and NMR relaxation. Front Phys. 2018;6:91.

56. Berry DB, Regner B, Galinsky V, Ward SR, Frank LR. Relationships between tissue microstructure and the diffusion tensor in simulated skeletal muscle. Magn Reson Med. 2018;80: 317-329.

57. Teh I, Zhou FL, Hubbard Cristinacce PL, Parker GJ, Schneider JE. Biomimetic phantom for cardiac diffusion MRI. J Magn Reson Imaging. 2016;43:594-600.
58. Huang SY, Nummenmaa A, Witzel T, et al. The impact of gradient strength on in vivo diffusion MRI estimates of axon diameter. Neuroimage. 2015;106:464-472.

\section{SUPPORTING INFORMATION}

Additional Supporting Information may be found online in the Supporting Information section.

FIGURE S1 Larger field of view SEM image showing the irregularity of the manufacturing process, including the large "extra-fibre" pores

How to cite this article: Huang C-CH, Hsu C-C, Zhou F-L, et al. Validating pore size estimates in a complex microfiber environment on a human MRI system. Magn Reson Med. 2021;86:1514-1530. https://doi.org/10.1002/mrm.28810 